\section{Allergologie in ganzer Breite}

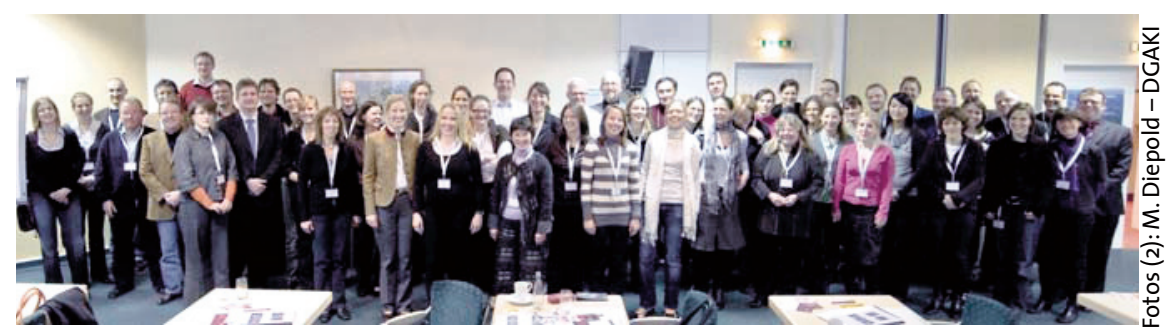

Die 1. Allergie-Akademie der DGAKI im Januar 2009 in Hannover war ein großer Erfolg. Mit dem von Dr. Ulrike Raap, Prof. Dr. Ulf Darsow und Dr. Helen-Caroline Rerinck aufgestellten Programm konnten insgesamt 77 Teilnehmer gewonnen werden.

D ie 1. Allergie-Akademie der DGAKI vom 22. bis zum 24. Januar 2009 war überschrieben mit „Angewandte Allergologie 2009 - Grundlagen und Praxis" und thematisch an das Curriculum Allergologicum (www.dgaki.de; Werfel et al. Allergo J 2000: 4; 202-5) angelehnt. Die Inhalte der Allergie-Akademie deckten die klinische Allergologie bezüglich aller relevanten Erkrankungen $a b$, angefangen von den Grundlagen (Begriffe, Definitionen) über Vorgehensweisen bei der Diagnostik/Untersuchung der Patienten bis hin zur Therapie. Damit stellt die Allergie-Akademie ein in Deutschland einzigartiges Angebot zur allergologischen Weiterbildung dar. Die Veranstaltung war mit insgesamt 23 Fortbildungspunkten akkreditiert.

Namhafte Referenten präsentierten Grundlagen und aktuelle Entwicklungen in der Allergologie mit hochinteressanten und interaktiven Fallvorstellungen. Die Qualität und das Niveau der Präsentationen waren erstklassig. Am

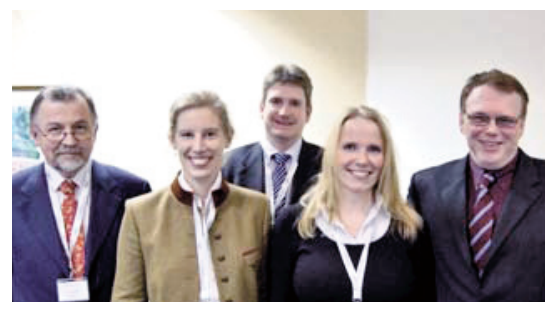

Die Organisatoren von rechts nach links: Prof. Dr. Claus Bachert (Präsident der DGAKI), Dr. Ulrike Raap, Prof. Dr. Ulf Darsow, Dr. Helen-Caroline Rerinck, Prof. Dr. Bernhard Przybilla (Referat Fortbildung der DGAKI)

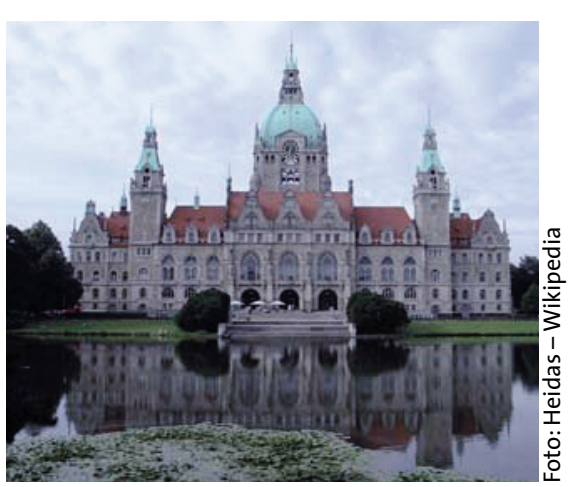

Der Veranstaltungsort: Hannover hat auch über die Allergie-Akademie hinaus einige Attraktionen zu bieten, wie hier das Neue Rathaus

Ende der Veranstaltung konnten alle Teilnehmer ihr neu erworbenes Wissen durch die Teilnahme am ersten DGAKIAllergologie-Akademie-Examen überprüfen, das von $90 \%$ der Teilnehmer bestanden wurde.

Das Feedback der Teilnehmer zur Akademie war exzellent. Das Willkommensdinner und der Gesellschaftsabend beim „Kanzler-Dinner“ boten eine gute Gelegenheit neue Freundschaften zu schließen und Kollaborationspartner kennenzulernen.

Das Organisationskomitee bedankt sich sehr herzlich bei allen Teilnehmern und den Sprechern für ihre aktive Teilnahme, die den Erfolg dieser 1. Allergie-Akademie der DGAKI ermöglicht hat.

Dr. Ulrike Raap, Hannover Prof. Dr. Ulf Darsow, München Dr. Helen-Caroline Rerinck, München

\section{Neue Öko-Tex ${ }^{\circledR}$-Kriterien in Kraft}

\author{
Die Öko-Tex ${ }^{\circledR}$ \\ Gemeinschaft \\ hat zum 1. Januar \\ 2009 die An- \\ forderungen der \\ textilen Schad- \\ stoffprüfungen \\ nach Öko-Tex ${ }^{\circledR}$ \\ Standard 100
}

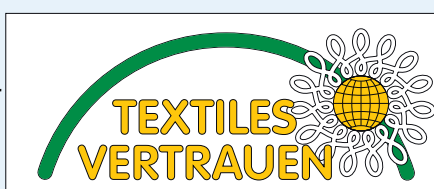
Schadstoffigeprüfte Textilien nach Öko-Tex Standard 100 Prüf-Nr. 00000000 Institut beispielsweise alle Grenzwerte und Prüfkriterien des Öko-Tex ${ }^{\circledR}$-Zertifizierungssystems ab sofort nicht mehr in ppm (parts per million) angegeben, sondern in der Maßeinheit $\mathrm{mg} / \mathrm{kg}$. Dadurch lassen sich mögliche Verwechslungen mit Volumeneinheiten wie etwa $\mu \mathrm{l} / \mathrm{l}$ oder $\mathrm{mg} / \mathrm{m}^{3}$ vermeiden. Weiter wurden verschiedene Chemikalien aufgrund einer geänderten REACh-Einstufung neu in die Liste der beim Öko-Tex ${ }^{\circledR}$-Standard 100 ausgeschlossenen Substanzen aufgenommen.

Die Öko-Tex ${ }^{\circledR}$-Schwermetall-Prüfungen auf Grundlage eines Eluats mit künstlicher Schweißlösung wurden um einen Totalaufschluss der beiden Substanzen Blei und Cadmium ergänzt. Damit ist es nun möglich, auch solche textilen Artikel zu überprüfen, in denen diese Schwermetalle in eine massive Matrix eingebunden sind und deshalb nicht anhand einer Schweißlösung exakt erfasst werden können: z.B. bei Deckfarben und Lacken im Spielzeugbereich, wo durch Alterung und mechanischen Gebrauch Farbstoffe mit Blei- oder Cadmiumgehalt absplittern und von Kindern verschluckt werden können. Hintergrund für die Aufnahme dieser beiden Prüfkriterien an der Schnittstelle Textil/Spielzeug sind die massiven Beanstandungen und Rückrufaktionen des letzten Jahres.

Neu im Öko-Tex ${ }^{\circledR}$ Kriterienkatalog ist darüber hinaus die Überprüfung auf Perfluoroctansulfonate (PFOS) und Perfluoroctansäure (PFOA). Da die europäischen Behörden aktuell noch eine allgemein gültige Prüfmethode ausarbeiten, verwenden die autorisierten Prüfinstitute der Öko-Tex ${ }^{\circledR}$-Gemeinschaft zunächst die von ihnen entwickelte Methodik zum Nachweis der zahlreichen als PFOS klassifizierten Einzelsubstanzen.

Die aktuelle Übersicht der Öko-Tex ${ }^{\circledR}$-Prüfkriterien und -Grenzwerte kann online unter www.oeko-tex.com/grenzwerte heruntergeladen werden. red 